\title{
Von Beruf Arzt und Kommunikator
}

\section{Charlotte Schweizer}

Leiterin Abteilung Kommunikation der FMH und Mitglied der Redaktion

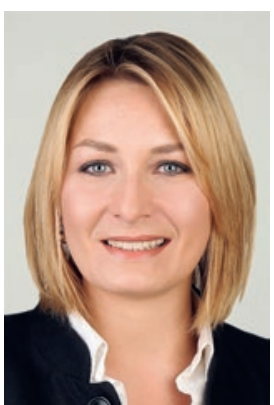

Als Kommunikationsleiterin der FMH weiss ich eines ganz genau: Wenn es um Kommunikation und Medienarbeit geht, dann ist jeder Spezialist. Jeder weiss, wie es funktioniert, und mit Sicherheit bekommt man noch ein paar Tipps, wie man alles ganz leicht besser und effektiver gestalten könnte. Dies nehme ich als Kompliment, denn es zeigt in erster Linie, dass die Kommunikation und Medienarbeit auf grosses Interesse stösst. Weiter zeugt der Raum, den die professionelle Kommunikation in vielen Gesprächen beansprucht, von der unbestrittenen Wichtigkeit, den die Kommunikation heutzutage einnimmt.

Was im hektischen Alltag von Ärztinnen und Ärzten fast vergessen gehen könnte: Im Unterschied zu vielen anderen stimmt es für Sie, liebe Ärztinnen und Ärzte, ganz bestimmt. Sie alle sind professionelle Kommunikatorinnen und Kommunikatoren. In Ihrem Beruf kommunizieren Sie ständig, und was Sie sagen, hat eine ganz entscheidende Wirkung. Tatsächlich ist es nicht nur, was Sie sagen, auch wie Sie es sagen, Ihre Wortwahl, der Gesichtsausdruck, der Ihre Worte begleitet, der Tonfall, Ihre Körperhaltung, Ihre Gestik, das alles ist Teil der Kommunikation. Nachweislich haben Sie als behandelnde Ärztinnen und Ärzte allein durch Ihre Kommunikation einen grossen Einfluss auf das Wohlergehen und den Therapieerfolg Ihrer Patientinnen und Patienten. Mit dem, was Sie sagen, können Sie Hoffnung geben oder aber Verzweiflung auslösen.

Die Bedeutung der Kommunikation in der medizinischen Praxis ist hinlänglich bekannt und wurde in zahlreichen wissenschaftlichen Studien belegt. So hat auch die Schweizerische Akademie der Medizinischen Wissenschaften (SAMW) der Kommunikation im medizinischen Alltag einen Leitfaden gewidmet. Darin erwähnen die Autoren als Folgen einer ungenügenden Kommunikation die «erhöhte Wahrscheinlichkeit einer Fehldiagnose, ein gestörtes Vertrauensverhältnis, das Anfordern unnötiger Tests und eine mangelhafte Compliance» [1]. Weiter gehört die Kommunikation zu den am häufigsten genannten Kriterien, die Patientinnen und Patienten während eines Spitalaufenthalts bemängeln (S. 8).

Es lohnt sich also, etwas genauer auf die eigene Kommunikation zu achten. Oft analysieren wir nicht, wie wir etwas ausdrücken und wieso. Manche Redewen- dungen haben wir aus Gewohnheit von unserem Umfeld übernommen. Wie häufig benutzen Sie zum Beispiel das Wort «eigentlich»? Und wie oft meinen Sie es wirklich? Worte haben ihre Wirkung, auch wenn uns das nicht immer bewusst ist. Und gerade wenn wir selbst Patienten sind und es um uns geht, dann hören wir plötzlich ganz genau hin. Was wäre Ihre Reaktion, wenn Sie von einer Ärztin hören würden, dass Sie eigentlich gute Genesungschancen haben? Ob eine Ärztin zu einem Bluthochdruck-Patienten sagt «Ich möchte gerne ein EKG machen, um sicher zu gehen, dass alles in Ordnung ist» oder ob sie sagt "Ich möchte gerne ein EKG machen, um zu schauen, wie stark ihr Herz geschädigt ist» - ziemlich sicher wird der Patient im einen und im anderen Fall die Tage bis zur Untersuchung mit anderen Gedanken zubringen. Über Kommunikationsstrategien denke ich jeweils auch nach, wenn mir Freunde erzählen, wie ein gewissenhafter Arzt seinen hoffnungsvollen Patienten mit beeindruckenden Statistiken vorrechnet, wie verschwindend klein ihre Chancen sind, jemals Eltern zu werden.

Diese Beispiele mögen übertrieben wirken, aber wie oft begegnen wir genau solchen Beispielen? Es gibt einen Trick bei der Kommunikation, der tatsächlich ganz einfach ist: Bevor der Sprecher eine Botschaft sendet, überlegt er sich kurz, wie diese bei ihm ankäme, wenn er der Empfänger wäre. Ja, es ist ganz leicht, und doch denken wir nicht immer daran.

Das folgende Sprichwort konnte nie zweifelsfrei einer Quelle zugeordnet werden; trotzdem möchte ich es zitieren, denn ich finde es wertvoll gerade auch in Hinblick auf die Kommunikation.

Achte auf Deine Gedanken, denn sie werden Worte.

Achte auf Deine Worte, denn sie werden Handlungen. Achte auf Deine Handlungen, denn sie werden Gewohnheiten. Achte auf Deine Gewohnheiten, denn sie werden Dein Charakter. Achte auf Deinen Charakter, denn er wird Dein Schicksal.

Achten wir uns doch alle in unserer täglichen Kommunikation etwas bewusster darauf, wie wir unsere Worte wählen und was wir dem Gegenüber mitteilen und mitgeben möchten. Und seien wir gespannt auf die Wirkung. 